\title{
A Study of the Outcome of the Latero-Central Glandular Pedicle Technique as an Oncoplastic Procedure for Medial Quadrants Breast Masses
}

\author{
Rania ELahmady, MD; Ahmed Gamal Eldin, MD; M.A. Marzouk, MD \\ Department of General Surgery, Ain Shams University, Egypt
}

Background: Numerous oncoplastic procedures are now available for treatment of breast cancer. Oncoplastic procedures had expanded the spectrum of breast conservative surgery. It is difficult to plan for resection of medial breast tumors as the scars are apparent and deformity is so eminent. The aim of the work is to assess the laterocentral glandular pedicle technique as an oncoplastic procedure for treatment of medial quadrant breast cancer as regard oncological safety, surgical outcomes and patient satisfaction.

Patients and method: This study included 15 female patients with a mean age of 43 years (20-60 years) and with invasive breast cancer at upper and lower medial quadrants. They were treated by wide in excision with safety margin followed by reconstruction of defects using latero-central glandular pedicle technique. The patients were followed for 6 months for postoperative complications and cosmetic outcome.

Result: In our study the mean age of the 15 patients was 43 years (20-60 years). $60 \%$ of the breast tumors were in the upper inner quadrant and $40 \%$ of them were in the lower inner quadrant. The largest tumor size was $3.2 \mathrm{cms}$, the smallest one was $1 \mathrm{~cm}$ and the mean tumor size was $2 \mathrm{cms} \pm 0.47$. The mean breast cup size for the whole study was $4.33 \pm 1.56$ which stand for $\mathrm{D}$. Our mean operation time was $107 \pm 14.50$ minutes ( 1 hour and 45 minutes). The longest post-operative stay period was 3 days. The postoperative complications occurred three cases $(20 \%)$ in the form of: 2 cases $(13 \%)$ of wound infection and 1case $(7 \%)$ of stitch sinus . none of the patients had recurrence during postoperative follow up period of six months duration.as regard the cosmetic outcome which was estimated via a score system graded from one to five, The overall mean score of our study was $4.23 \pm 0.86$ which falls between very good and excellent.

Conclusion: The latero-central glandular pedicle technique is an oncoplastic procedure for treatment of medial quadrant breast cancer with main advantages of low rate of postoperative complications and better cosmetic outcome.

Abbreviations: Nipple-areola complex (NAC), Multidisciplinary team (MDT).

\section{Introduction}

Breast cancer is the most common site of cancer in women in Egypt according to national cancer institute, as it accounts for about $32.8 \%$ of total malignancies among Egyptian females; it is one of the important causes of mortality among women. ${ }^{8}$

Oncoplastic techniques are surgeries that aim to combine the principles of oncologic resection and reconstruction at the same time. so, wide excision with safety margin can be achieved without the risk of deformity. ${ }^{7}$

Oncoplastic surgeries had gained popularity using several pedicles for nipple-areola complex. The type of surgery can be tailored according to tumor situation. The most common pedicles for medial quadrant tumors are superior and inferior pedicles. ${ }^{5}$ The superior pedicle technique gives the surgeon the ability for excision of the breast tumors in the central and inferior pole of the breast, also tumor located in the medial or lateral quadrant as well as retroareolar tumor on condition that the tumor is not closed to the skin. This type of mammoplasty is difficult to be used in small non ptotic breast (an areola to inframammary distance has to be more patients who underwent superior pedicle mammoplasty suffer from decreased sensation at the nipple-areola complex within 1 year irrespective to the amount of the tissue resected.

The inferior pedicle mammoplasty allows well perfusion of the nipple-areola complex in the breast of any size and shape. It can be performed in 2-3 hours however it is an easy procedure, it takes this time as it needs some flap undermining. The inferior location of this procedure gives it the advantage of lower wound complication rat as it obliterates the dead space in the dependents part of the breast. The development of "bottoming out" phenomenon is the major disadvantage of this technique. ${ }^{9}$

The major advantage of the lateral pedicle technique over superior pedicle technique in large breast is the avoidance of pedicle kinking. In lateral pedicle procedure branches from the lateral thoracic 
artery add to the blood supply and deep branches of the fourth intercostal nerve are contributed to sensation, so this technique is characterized by good arterial input and excellent results in the preservation of nipple-areola complex sensitivity and is highly suggested for young female patients in whom preservation of nipple sensitivity is of great important. ${ }^{4}$

Flattening of the nipple-areola complex in lateral pedicle procedure is avoided by the glandular tissue which present behind the nipple-areola complex supporting it, so improves projection and the conic appearance of both the breast and the nipple. ${ }^{2}$

\section{Aim of the work}

The aim of our work was to evaluate of the laterocentral glandular pedicle technique as an oncoplastic procedure for treatment of medial quadrant breast cancer as regard oncological safety, postoperative complications and cosmetic outcome.

\section{Patients and methods}

This prospective study was conducted on 15 patients diagnosed to have early stages of breast cancer in between 2017 and 2018 at General Surgery Department in Ain Shams University Hospitals with approval of the Ethical Committee and written informed consent was obtained from all participtans. Patient selection was done through inclusion and exclusion criteria.

\section{Inclusion criteria:}

- $\quad$ Female patients with age ranging from 20-60 years.

- Patients with invasive breast cancer at upper and lower medial quadrants.

- Patients whom oncoplastic surgery for both inner quadrants of breast were possible on oncologic grounds.

- $\quad$ T1-T2 node positive patients(TNM classification).

\section{Exclusion criteria:}

- Age less than 20 years.

- Patients not convinced with the procedure after adequate explanation.

- Distant metastasis.

- History of previously treated ipsilateral breast cancer.

- Absolute contraindication for postoperative adjuvant chemotherapy.

- T3 and T4 breast cancer.

- Lesions involving or less than $1.5 \mathrm{~cm}$ from nipple and areola.

- Multicenteric breast cancer.

- Inflammatory breast cancer.

Patients were subjected to Clinical assessment (history, full breast and axillary examination), investigation (routine laboratory investigations, bilateral sono-mammography, histopathological examination for suspicious breast mass, pelviabdominal u/s, CT chest and bone scan.

\section{Operative technique}

With the patient standing in an upright position measurements were taken before receiving preanesthetic drugs. Drawings were done using water proof skin markers.

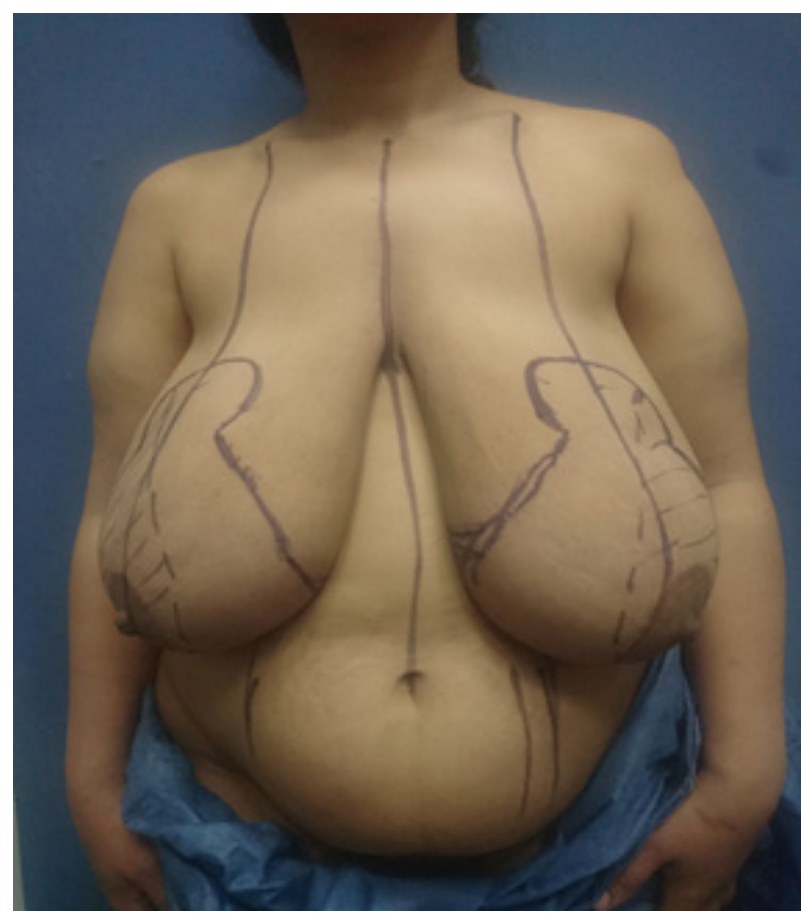

Fig 1: Preoperative drawings while the patient standing.

Then the patient was placed in the supine position with the arms abducted for axillary access, with the possibility to seat the patient on the operative table to control the symmetry.

A reduction mammoplasty keyhole pattern incision was made, and then de-epithelialization of the area on top of the glandular pedicle between the new NAC and the lateral vertical incision was done.

A full thickness incision was performed at the border of the new areolar position and the medial and lateral incision lines that converge at the inframammary crease.

A subcutaneous undermining was performed to the inframammary crease in order for preparation of the inferior horizontal resection. In very large breasts, prepectoral undermining of the breast gland was performed in the lower $2-3 \mathrm{~cm}$. This was done to allow easy removal of the lower excess of breast tissue.

The next step was the creation of a lateral glandular pedicle. In order to preserve the lateral 
vascularization and innervation of the nipple-areola complex, a column of glandular tissue between the nipple-areola complex and the pectoralis muscle was created in continuity with the lateral pillar of the breast. The base of this column is situated on top of the pectoralis muscle to include the pectoralis muscle perforators and is not detached from the muscle in the process of gland resection. To avoid this, the breast gland should be relocated and held by the surgical assistant in a more medial and cranial position as it is shifted laterally as the patient lay down.

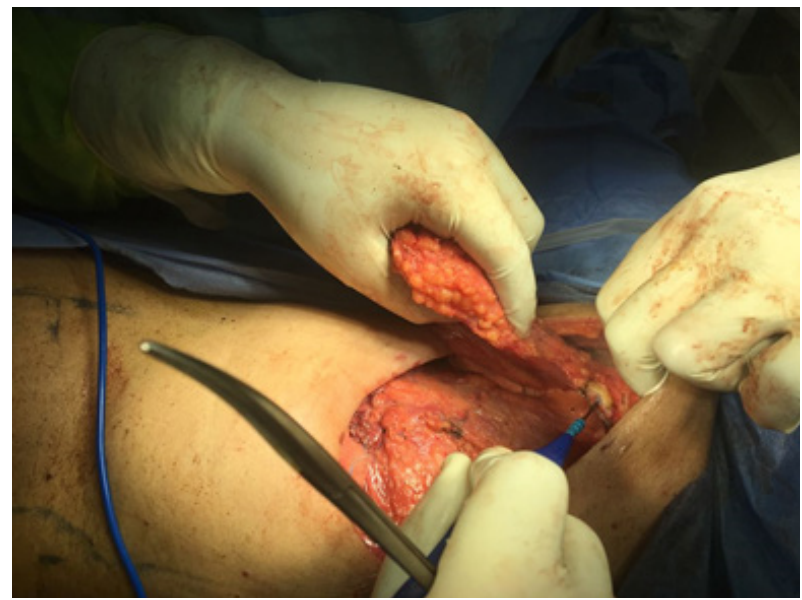

Fig 2: Intra-operative dissection.

Along the lines of the lateral glandular pedicle, drawn pre-operatively, and around the medial edge of the NAC, a straight incision was made down to the pectoralis muscle. Just before reaching the pectoralis muscle, the incision can be beveled outwards to increase the number of perforators included into the pedicle.

As regard the tumor full thickness excision of the lesion was completed, with at least a $1 \mathrm{~cm}$ macroscopic margin of normal tissue and the skin overlying the lesion was removed.

Following tumor resection and intraoperative margin assessment via frozen section examination, the cavity was clipped for radiation boosting and postoperative surveillance.

Then the remaining breast tissue was examined. Additional tissue should not be resected until it has been detected if the dead space can be filled with the lateral pedicle or not.

The horizontal resection in the lower poles of the breast should be performed after the creation of the columnar lateral glandular pedicle in order to avoid deformation of the glandular pedicle during incision. The horizontal resection was performed by making a straight cut down to the pectoralis fascia along the horizontal line drawn preoperatively.
Next step was, the lateral and medial pillars were created. The lateral incision down to the pectoralis muscle will be in continuity with the inferior incision of the glandular pedicle. The medial incision down to the pectoralis continues into the area cranial to the lateral glandular pedicle.

The area between the superior border of the lateral glandular pedicle and the new nipple areola complex was removed following the lines of the preoperative drawings. All glandular tissue between the skin and the pectoralis muscle was resected in a conical fashion to create a cylindrical cavity that will accommodate the columnar shape of the lateral glandular pedicle.

To creat a lateral pedicle flap, infra-mammary incision was done and undermining of the breast tissue off the pectoral fascia to mobilize the nipple areola complex and underlying tissues. Mobilization of the breast tissue helps in palpation of both the deep and superficial surfaces of the tumor, which can aid in determining the lateral margins of excision around the target lesion. The keyhole pattern can be rotated slightly to allow for a more lateral or medial excision, while at the same time, the nipple areola complex was moved in a direction opposite to that of the surgical defect.

The lateral glandular pedicle with the areolar complex was repositioned. The entire column pivots on the perforators of the pectoralis muscle and the nipple areola comlex was rotated cranially to fit in its new position. To avoid kinking or compression of the pedicle the glandular pedicle was only kept in place by subdermal suturing.

Both pillars were sutured together with separate deep and superficial resorbable stitches to provide a nice conical shape of the breast. We should avoid closing the pillars too tight to avoid compression of the glandular pedicle.

Adequate hemostasis was done with application of surgical drains.

The skin was sutured according to the incision if it was a vertical incision only or converted into an inverted $\mathrm{T}$.

The decision to perform only a vertical scar or an inverted " $T$ " scar depended on the level of hypertrophy and the level of ptosis. A vertical scar only was performed small breasts and those with less ptosis, and an inverted " $T$ " scar for large breasts with major ptosis avoiding the cutaneous excess such as the skin fold produced in the vertical scar. The position of vertical or an inverted "T"scar can be central (more frequent), medial, or lateral according to the location of the tumor and the need 
for skin removal.

Skin closure was performed by subdermal resorbable suturing; Aseptic dressings, skin adhesive and a supportive bra were put on.

A subcutaneous absorbable 3-0 suture was placed around the areola at a size of the original NAC, and then 4-0 subcuticular sutures were used to close the wound.

The same procedure were performed in a mirrorimage fashion during the same operation time for the contralateral breast

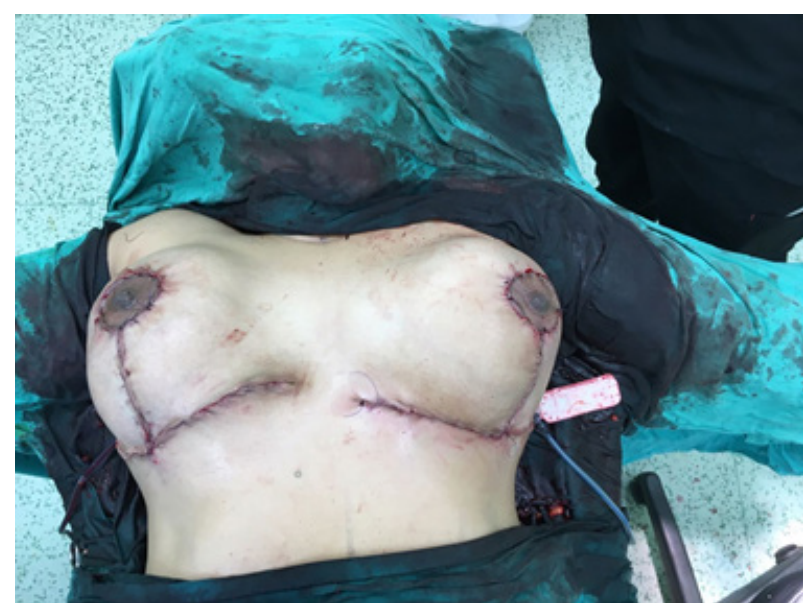

Fig 4: Postoperative wound closure.

An axillary lymph node dissection was done.

Prophylactic broad-spectrum antibiotic was given to all patients upon induction and during the whole period of hospital stay.

The patients were given routine post-operative analgesia in the form of Nalofen $50 \mathrm{mg}$ after recovery from anesthesia followed by NSAIDs fixed dose every 12 hours in the first 24 hours and when needed after that.

If no complications developed patients were discharged at the third day post-operative on antibiotics, analgesics and anti-edema agents, and they were advised to wear well-fitting sport bra.

Drains were removed when daily volume is minimal. To avoid stiffness of the shoulder joint and decrease arm edema after axillary surgery, Patients were instructed to undergo arm and shoulder mobilization and a set of exercises

A follow up schedule was given to the patients as the following first week every three days for dressing, the following two weeks every four days until removal of the stitches.
Our patients were followed up for both oncologic and cosmetic grading and were referred to receive postoperative suitable adjuvant chemo and or radiotherapy according to the final pathology reported after conventional pathological evaluation. Patients were asked to follow up in the surgical department clinic after completion of the adjuvant therapy once every three months for the six months.

\section{Results}

Our study included 15 female patients with invasive breast cancer who underwent oncoplastic breast surgery via two major technical steps, excision of the tumor with wide safety margin along with formal axillary dissection, followed by immediate reconstruction using lateral pedicle technique.

The age of the patients varied from 20-60 years old (mean age was 43), three patients had medical comorbidities. 1 patient had diabetes mellitus, 1 patient had hypertension and 1 patient had ischemic heart disease.

The tumor size was evaluated by ultrasound, the smallest tumor size was $1 \mathrm{~cm}$ and the largest was 3.2 $\mathrm{cm}$ with mean tumor size $2 \mathrm{~cm} \pm 0.47$. The breast cub size was estimated and score was given, the mean breast cup size for the whole study was $4.33 \pm 1.56$ which stand for D (Table 1). The minimum breast cup size was $C$ and the maximum was $G$. The nearest tumor was $1.5 \mathrm{~cm}$ from NAC and the farthest was $4.6 \mathrm{cms}$ from NAC with mean distance 2.84 \pm 0.93 . The tumor was in the upper inner quadrant in $60 \%$ of the patients and in the lower inner quadrant in $40 \%$ of the patients.

Table 1: Breast cup size score given for the case in the study

\begin{tabular}{lc}
\hline Breast cup size & Given score \\
\hline Breast Cup Size A & 1 \\
Breast Cup Size B & 2 \\
Breast Cup Size C & 3 \\
Breast Cup Size D & 4 \\
Breast Cup Size E & 5 \\
Breast Cup Size F & 6 \\
Breast Cup Size G & 7 \\
\hline
\end{tabular}

Our mean operation time was 107.60 minute \pm 14.50 , the fastest procedure was in 90 minutes and the longest took 155 minutes. No patient needed blood transfusion neither intra or postoperative. Most of the patients were discharged 24 hours postoperative the mean postoperative stay period was 30 hours \pm 8.77 ; however the longest stay period was 3 days.

Postoperative complications occurred only in 3 
cases in the form of two cases of wound infection and one case of stitch sinus. The 2 patients with infection received oral antibiotics and instructed to have a daily twice wound dressing until the infection was eradicated. One of the 2 only required wound opening to allow adequate drainage and then secondary suture was done.

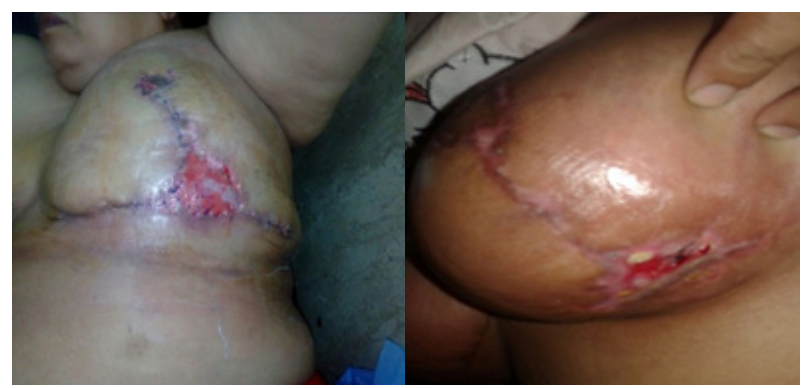

Fig 5: Postoperative infected wounds.

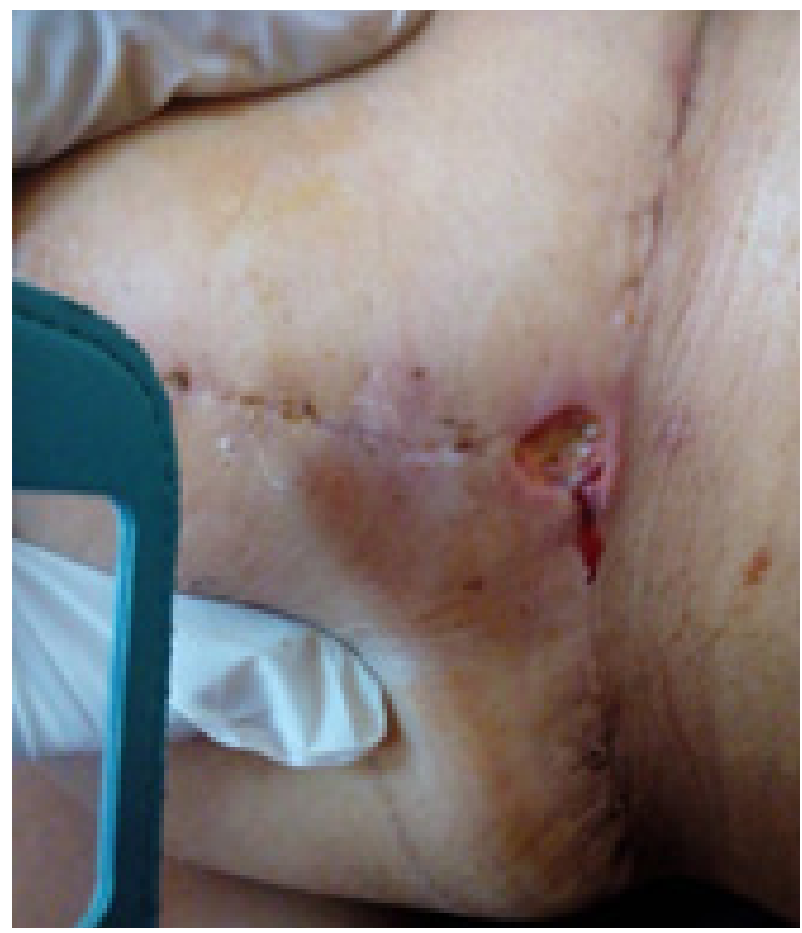

Fig 6: Postoperative stitch sinus formation.

As regard post-operative pathological outcome 11 of our patients had IDC, 3 of our patients had ILC and one of them had mucinous carcinoma. All our patients had free margins in frozen sections taken during the operation and none of them had recurrence or needed further surgery during postoperative follow up period of six months duration.

Cosmetic outcome was evaluated using score system graded from 1 to 5 (Table 2) it was made up by surgeon, patient and breast MDT using photographs taken 2 weeks and one month. It based on multiple items:

1. The overall shape of the breast.
2. The symmetry of both breasts.

3. The site and direction of the nipple.

4. The volume if the breast.

5. The skin incision shape.

Table 2: Postoperative cosmetic scoring system

\begin{tabular}{ll}
\hline 5 & Excellent \\
4 & Very good \\
3 & Good \\
2 & Fair \\
1 & Poor \\
0 & Ugly \\
\hline
\end{tabular}

In our study the overall mean score was 4.23 which was between very good and excellent (Table $\mathbf{3}$ ). 10 cases gave excellent score (score 5), 3 cases gave very good score (score 4), 1 case gave good score (score 3), 1 case gave fair score (score 2), 0 case gave poor score (score 1 ) and 0 case gave ugly score (score 0).

Table 3: Mean cosmetic outcome for our study

\begin{tabular}{ccccc}
\hline & Mean & 土SD & Minimum & Maximum \\
\hline Cosmetic outcome & 4.23 & .86 & 2.00 & 5.00 \\
\hline
\end{tabular}

\section{Discussion}

Oncoplastic surgery is the combination of oncologic and cosmetic surgery. This "third pathway" had allowed extending the indications of breast conserving surgery without compromise of oncologic goals or the esthetic outcome. There are three basic principles on which oncoplastic breast surgery is based: Ideal breast cancer surgery with more wide excision, immediate symmetry of the other breast whenever necessary and immediate breast reconstruction. ${ }^{7}$

A bilateral reduction technique is the most appropriate approach in women with Brassiere cup size C, D or larger with coexisting ptosis. It provides the most desired oncological outcome with attention to the cosmetic result. Also, it results in a higher patient satisfaction rate due to their perception of improved aesthetic outcomes. Paridon et al. 2017 published that the cosmetic outcomes of oncoplastic breast reduction assessed through BREAST-Q surveys that were completed by the patients, they were satisfied with their breasts at $(72.6 \% \pm 20.4)$ and also satisfied with their outcome $(74.6 \% \pm 17.6){ }^{13}$

We were able in our study to conduct an excellent cosmetic outcome for a relatively large tumor excisions as $66.6 \%$ of the cases (10 patients) was falling in excellent and very good score groups with mean cosmetic outcome score 4.23. The other $33.3 \%$ ( 5 cases) was falling in good and fair score 
groups this was because of the two patients who noticed asymmetry of the two breasts in front of the mirror as they refused bilateral breast reduction mastopexy. None of our cases were in the ugly score group.

After reduction mammaplasty changes of nipple and areola sensitivity do not depend on the weight of resection. But they depend on the surgical technique. The superior glandular pedicle technique with tissue resections at the base of the breast is associated with a higher risk of injury to the nerves supplying the nipple-areola complex. Although sensitivity starts to improve between 3 and 6 months after surgery, it does not recover fully even 12 months after the operation. ${ }^{14}$

As regard nipple and areola sensitivity, it was found by Schlenz et al. 2005, that $47.8 \%$ of the patients who underwent operation by superior pedicle techniques three weeks postoperatively had insensate nipples. Most of the insensate nipples were bilateral $(72.7 \%)$. The sensitivity of the nipple was unchanged in patients who underwent operation with lateral or inferior pedicle technique. In our study, all cases sensitivity started to improve within three to six months nearing the preoperative values. ${ }^{14}$

In order to reduce the cancer recurrence rate obtaining clear margins (the larger, the better) is an essential step in the procedure. The recurrence rate is mainly influenced by the tumor size, the tumor type, multicentric disease vascular invasion, and the age of the patient.

Annual recurrence curves indicated that the relapse peak after mastectomy mainly emerged in the first two years; however, after conservative breast surgery recurrence increased annually with the highest peak near five years as shown by most of the studies. ${ }^{3}$

In our study there was no malignant recurrence as confirmed by the pathologist in our multidisciplinary team. The follow up period of the malignant recurrence was the first six months as this was the time limitation of our study.

The operative duration of our study was at first long (155 minutes) but then with progression of our study, the operating time was reduced to (90 minutes) within an average of (134 minutes). This was in comparison to the average of (120 minutes) by Blondeel et al., 2003.

In our study only three cases (20\%) have had complications. two of them had wound infection and one had stitch sinus. One of the 2 cases with wound infection was diabetic reflecting the immune compromisation with diabetes mellitus. Statistically DM has increased the risk of post-operative wound infection three times, this similar to what was published by Urban C and Rietjens M, 2013 showing the complication of diabetes mellitus in oncoplastic surgery.

We reported lower results of surgical wound infection (13\%) than reported by Vilar-Compte et al., 2009 (18.9\%) and higher than reported by Olsen et al., 2008 (4.7\%). Also, even if there was a complication of surgery no cases were delayed in delivery of postoperative loco-regional irradiation or adjuvant chemotherapy.

\section{Conclusion}

The choice of the oncoplastic technique is mainly based upon the size of the breast, location of the tumor, and distance of the tumor from the nipple areola complex. So, all cases should be reviewed adequately in order to tailor the decision for every single case.

With calculation of the risk factors for recurrence and postoperative complications, oncoplastic surgery with the lateral pedicle breast reduction technique is considered a good option for patients with large breasts and node-positive breast tumors in both inner quadrants. Especially, if we take in consideration the advantages of this technique, which are, the increase of the viability of the pedicle ,the preservation of sensation in nipple areola complex and the support by glandular tissue behind the nipple areola complex which improves projection and hereby the aesthetic outcome of both breast and nipple.

\section{References}

1. Blondeel PN, Hamdi M, Van de Sijpe KA, et al: Thelatero-central glandular pedicle technique forbreast reduction. British Journal of Plastic Surgery. 2003; 56(4): 358.

2. Chiummariello $S$, Angelisanti $M$, Arleo $S$, et al: Evaluation of the sensitivity after reduction mammoplasty. Our experience and review of the literature. Ann Ital Chir. 2013; 84: 385-388.

3. Clough K, Lewis J, Couturaud B, et al: Oncoplastic techniques allow extensive resections for breastconserving therapy of breast carcinomas. Ann Surg. 2003; 237(1): 26-34.

4. Fitzal F, Nehrer G, Deutinger M, et al: Novel strategies in oncoplastic surgery for breast cancer: Immediate partial reconstruction of breast defects. Eur. Surg. 2007; 39/6: 330-339.

5. Hamdi M, Hall-Findlay EJ: Vertical Scar Mammaplasty. Pedicle Choices in Breast 
6. Hau E, Browne L, Capp A, et al: The impact of breast cosmetic and functional outcomes on quality of life: Long-term results from the St. George and Wollongong randomized breast boost trial. Breast cancer research and treatment. 2013; 139(1): 115-123.

7. Inas $A$, Nelly $H$, Manar $M$, et al: Cancer epidemiology and biostatics, NCI Cairo cancer registry Egypt. Breast cancer. 2008: 45-46.

8. Jones A, Pu L: Oncoplastic approach to early breast cancer in women with macromastia. Ann Plast Surg. 2007; 58(1): 34-38.

9. Khaled $\mathrm{H}$, Mikhail $\mathrm{N}$, Kamel $\mathrm{H}$, et al: Cancer Incidence in Egypt: Results of the National Population-Based Cancer Registry Program. Journal of Cancer Epidemiology. 2014; 18.

10. Kronowitz S, Feledy A, Hunt K, et al: Determining the optimal approach to breast reconstruction after partial mastectomy. Plast Reconstr Surg. 2006; 20107(1): 1-2010.

11. McCulley SJ, Macmillan RD: Therapeutic mammaplasty: Analysis of 50 consecutive
12. Olsen M, Chu-Ongsakul S, Brandt $E$, et al. "Hospital-associated costs due to surgical site infection after breast surgery," Archives of Surgery. 2008; 143(1): 53-60.

13. Paridon MW, Sloane $P$, Amendoeira I, et al: Oncoplastic breast surgery: Achieving oncological and aesthetic outcomes. 2017; 195202.

14. Schlenz I, Rigel S, Schemper M, et al: Alteration of nipple and areola sensitivity by reduction mammaplasty: a prospective comparison of five techniques. Plast Reconstr Surg. 2005; 115: 743-751; discussion 752-754.

15. Urban C, Rietjens M: Oncoplastic and Reconstructive Breast Surgery. Springer-Verlag Italia, $1^{\text {st }}$ edition. 2013: 13-20.

16. Vilar-Compte D, Rosales S, Hernandez-Mello N, et al: "Surveillance, control, and prevention of surgical site infections in breast cancer surgery: a 5-year experience," American Journal of Infection Control. 2009; 37(8): 674-679. 